\title{
Developing a Provincial Centralized Intake Process for a Knowledge Resource Service Part 1: Literature Search Requests ${ }^{1}$
}

\author{
Marcus Vaska, Elizabeth Aitken, Janice Varney, and Spencer Stevens
}

\section{Introduction}

In 2009, the Alberta government amalgamated nine regional health authorities and three provincial services [1] into one entity with five zones, creating Alberta Health Services (AHS). AHS is responsible for providing and coordinating a province-wide health care system to serve 3.8 million Albertans. AHS includes almost 100,000 staff members, 8000 plus physicians, over 400 sites, and more than 32,000 beds [2].

Prior to the formation of AHS, each region had developed its own strategy for providing library services. For example, Calgary Zone outsourced library services to the University of Calgary, whereas the other regions created their own local services, collections, and staffing [3]. Since 2009, library services have been gradually drawn together into a team that serves the entire province of Alberta. This new Knowledge Resource Service (KRS) is part of the provincial Knowledge Management (KM) department within Alberta Health Services. It is comprised of library teams and KM consultants located across the province. KRS connects AHS staff and healthcare providers with evidence-based resources and supports them in effectively using these resources to make evidenceinformed decisions and to promote quality patient care.

As we worked toward becoming a provincial team providing equitable access to resources and services to all AHS staff, it became apparent that the ways we were offering service needed to evolve. The Research and Reference work group, as part of the KM department, was formed in 2012 as a standing virtual team of librarians, library technicians, and information specialists within KRS. Its ongoing goal is to create, develop, implement, and evaluate research and reference services for the organization. In 2013, the top priority of KRS was to develop a single point of intake for receiving and processing literature search and document delivery service (DDS) requests. As this is part one of a two part program description, the DDS request service will be discussed in a forthcoming issue.

Based on a review of the literature, we determined that a single point of intake for more than 100,000 health care practitioners had not been previously described. Although there is considerable literature discussing triage in the hospital emergency setting, far less has been written on standards for managing digital reference requests. Pomerantz [4] suggests that the process of triaging incoming requests requires very clear and tangible criteria to make it work successfully. Others, such as Lyon et al. [5] look at other technical approaches to capturing similar reference data. We discuss our experiences in how to make this single intake process work in a distributed virtual team environment.

Our objectives were to: $(i)$ maximize the provincial workflow by developing a single point of intake for receiving and processing literature search requests; (ii) provide a tool for sharing information about the breadth, scope, and trending topics of literature searches; and (iii) track the collective work effort for workload, efficiency, and equitable delivery of high-quality service.

\section{Description}

As can be seen from the 3000 literature search requests received between August 2013 (the date a new provincewide KRS website was launched) and May 2014, literature

\footnotetext{
Marcus Vaska². Knowledge Resource Service, Health Sciences Library, Rm 1491B, 3330 Hospital Drive NW, Calgary, Alberta, T2N 4N1.

Elizabeth Aitken. Knowledge Resource Service, Rm. 4EE11, 700714 St. SW, Calgary, Alberta T2V 1P9, Rockyview General Hospital.

Janice Varney. Knowledge Resource Service, Rm. 111, 6665 St SW, Medicine Hat, Alberta, T1A 6L4, Medicine Hat Regional Hospital.

Spencer Stevens. [retired] Knowledge Resource Service, Rm. A2-908, 2888 Shaganappi Trail NW, Calgary, Alberta T3B 6A8, Alberta Health Services, Alberta Children's Hospital.

${ }^{1}$ This article has been peer-reviewed.

2Corresponding author (e-mail: mmvaska@ucalgary.ca)
} 
searching is a service in heavy demand; therefore, the creation of a single point of intake was assigned a top priority by KRS management. Following an environmental scan of current processes surrounding literature search queries, (i.e., a search of the current literature as well as numerous discussions among colleagues province-wide regarding processes followed at their institutions), a literature search intake process and triage document was visually mapped out by the Research and Reference Workgroup.

The literature search intake and triage process was created to track the number and locations of literature searches conducted in each provincial zone and to maximize enterprise-wide workflow. It has also served as a tool for sharing information about the breadth and scope of literature searches requested on a service-wide scale. Due to the magnitude of developing a single point of intake for receiving and processing literature search requests, a pilot project commenced in January 2013. Originally intended to run until 31 March 2013, due to technical challenges it was necessary to extend the pilot study for an additional three months, concluding on 30 June 2013. Using an Excel spreadsheet (Figure 1), each element of each literature search was meticulously documented including requestor, topic question, searcher, time spent, and resources consulted. We looked at e-tools that might help deliver this in an integrated fashion, and in concert with the Web workgroup, LibAnswers (an online reference platform from Springshare) was selected. It automatically populates any information received via the online request form, and telephone, fax, e-mail, and in-person requests were entered manually. These latter methods rapidly decreased in popularity as clients became accustomed to the new website, so the extra work for data entry was negligible. Overall, this pilot project was effective in helping us determine the elements we needed to capture in forms and reports.

During the pilot project, three triage decision criteria elements were applied to each and every literature search request received: the urgency of the request, the depth of the search, and the subject matter of the question.

The first criterion was urgency. The online request form asks requestors to differentiate urgent patient care questions from standard requests. In a healthcare environment, patient care is a top priority; therefore, urgent clinical patient care requests are processed on the same business day received.

The second element is how comprehensive the search needs to be. Finfgeld-Connett and Johnson [6] state that transparency of a search, no matter how complex, "enhances integrity of knowledge-building". Each litera- ture search request was assigned one of three degrees of complexity: quick (time-limited, often consisting of one research database), standard (more time involved, at least two databases consulted, with grey literature also being considered), and extensive (complex literature searches, carried out over many months, involving multiple search strings and resources (databases and grey literature)). Extensive literature searches may also lead to the researcher receiving acknowledgement for his/her efforts in the project or being included as a co-author. The KM department is integral to the work of many strategic clinical network initiatives, so this level of searching is quite common.

The third element is the question topic. KRS librarians and library technicians who have developed subject expertise were asked to self-identify as having experience in conducting literature search requests in one of the following disciplines: cancer, mental health, rehabilitation, pharmacy, pediatrics, and public health. Requests of an extensive nature were referred to the appropriate searcher based on the subject area expertise indicated. In cases where there was no subject expert identified, the question was forwarded to all searchers for consideration.

In an effort to develop a seamless process from receipt of literature search request to delivery of search results to the client, a gatekeeper model was created. The gatekeeper was assigned to triage requests according to level of complexity, referring to a subject search specialist when required, as well as to ensure that each search was picked up by a searcher within 24 hours of request submission.

Program development was ongoing throughout the course of the pilot study, with a number of alternative options being explored and previous proposals discarded. For instance, the gatekeeper approach originally involved a two-person buddy system, with one gatekeeper (and a back-up) solely responsible for monitoring literature requests over a one week period. The gatekeeper role was to ensure that priority requests were handled promptly, outstanding literature search requests were claimed within a 24-hour period, and extensive subject-specialty requests were relayed to the appropriate subject librarian/information specialist. The gatekeeper role was a responsibility held exclusively by a library technician or information specialist. In AHS KRS, the information specialist is a library technician role responsible for reference, document delivery services, and some literature searching. The intent of this decision was to free up the librarians' time from having to monitor the various communication intake means (e-mail, phone, fax, in-person) so they could concentrate on working with clients on identified complex searches and build positive relationships with key

Fig. 1. KRS literature search tracking excel spreadsheet.

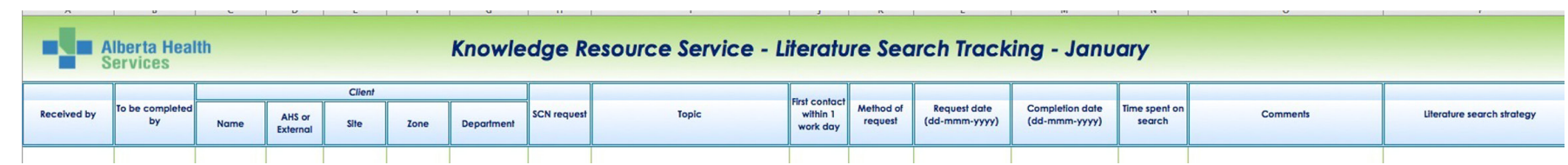


stakeholders, faculty members, physicians, clinicians, and researchers within AHS.

\section{Outcomes}

After the initial trial period, focus groups were conducted with all library staff who participated in the pilot study, and a number of changes were implemented as a direct result. The practice of gatekeepers referring complex requests to subject specialists was quickly abandoned. The reason for this was three-fold: (i) seemingly complex requests could usually be handled by any KRS staff member trained in literature searching processes, and on the rare occasions that a request truly required the participation of a searcher experienced in that particular discipline, it could be referred or help requested; (ii) it slowed down the referral and search process; and (iii) it discouraged searching staff from challenging themselves and gaining expertise in a new topic area. We found a 24-hour claim time to be very demanding and moved it to 48 hours (except for urgent requests), adjusting our suggested timelines for results delivery to five days or a client-specified date. Those deadlines are often renegotiated when we do the reference interview. The gatekeeper role was rolled into more of a queue-monitoring function combined with a chat reference service to be sure searches were picked up - but not to assign them. Claiming searches continues to be self-directed.

The confidentiality of patrons' requests was discussed. This is an internal database that will not be made accessible to anyone outside of the KRS unit. LibAnswers provides the capability of creating public answers, but they would be stripped of requestor detail and used only as an FAQ if we could be reasonably certain the topic would not identify the requestor. If a client has concerns about their name appearing anywhere (for example in a challenging Human Resources or medical error situation) the question can be blinded.

As a result of lessons learned regarding the delivery of literature search results to the client, an additional change was implemented. Some staff already used a template to ensure that search results were returned in a consistent manner to clients. This was a great opportunity to create a branded product and a search description template outlining resources used, strategies, limits, and links to the e-resources and document delivery services. It had the internal benefit of standardizing the elements reported for improved follow-up, providing a more professional appearance, and creating a solid start to a service standard.

This has been an excellent initiative for our new enterprise-wide literature searching team. The introduction of LibAnswers as a tool has been a major undertaking, yet it is well suited to our multisite environment. From a management perspective we have access to a rich source of information to monitor services and inform our planning. We are able to:

- track the amount of time spent searching and become more globally aware of the types of questions being asked and by whom,
- monitor use of print and electronic resources to guide collection decisions,

- identify gaps or needs for staff development,

- monitor response time, and

- share the work equitably across the team with decreased response delays due to staff vacations or illness.

\section{Discussion}

"The whole is greater than the sum of its parts," a quote often attributed to Aristotle, truly inspired the creation of the team-oriented centralized intake process for fielding literature search requests. Based on an extensive environmental scan of existing literature search practices throughout AHS, as well as a literature review looking at triage methods in hospital settings, there were several frameworks that were applied in creating the current KRS literature search request model. As of this writing, a number of steps have already been taken to improve this service in the coming year. An internal Community of Practice has been developed for literature searchers within KRS, enabling them to share in a collegial and peersupported atmosphere and providing tips, tricks, learning opportunities, and ideas for dealing with challenging searches. Staff meets virtually by means of Lync meetings and a blog. Following consultation with KRS literature searchers and managers in June 2014, Literature Search Service Standards were implemented to ensure consistency in turnaround time, accompanying search details, communication methods, and appearance of the search results. These standards are listed on the KRS website, http://krs. libanswers.com/a.php?qid $=581791$. Finally, in July 2014, a literature search training program for all KRS staff was launched to teach new skills, review current literature search patterns, and establish a more consistent means of responding to client requests on an ongoing basis.

The numerous accolades received by AHS KRS staff since the literature search service was launched last year have been very encouraging, but there are a few limitations that need to be addressed. Long-established recommendations require a searcher to acknowledge receipt of a client's request within 48 hours, a practice that is followed for the most part but can be inadvertently forgotten during times of high volume. Further, standard turnaround times for delivery of preliminary results may need to be adjusted or an additional category considered, especially when requests are deemed to be urgent but do not involve direct patient care. Finally, a more rigorous gatekeeper check method may need to be employed at times when requests are not claimed within the standard 48-hour period.

\section{Conclusion}

At the time of submission of this program description, the Knowledge Resource Service has passed its one-year anniversary of providing a single point of intake for literature search services to AHS staff located in all corners of the province. Although there have undoubtedly been some challenges and subsequent changes in practice over 
the past year, it has been an interesting journey. We know that our client group is very ready to embrace an online service request alternative. We have developed a queue monitoring process that is automated, gathered statistics and information across a large physical area with many sites, and shared the workload. The pilot project gave us the opportunity to test ideas such as specialized searching expertise and controlled gatekeeping. We now know that eliminating potential bottlenecks and not micromanaging the request stream works for us. Time will tell if that remains true in times of very high volume. The secondary value was that the group effort greatly increased our ability and desire to build a strong virtual team. The single point of entry for literature search requests has been well received, with clients commenting that having the ability to request services at any time from any location is a remarkable feat. There has been a noticeable increase in uptake from rural areas as a result. Future analysis of the accumulated data will permit us to continue to revamp our literature search services. Our collaborative virtual team will work to establish new core standards and modify practices to meet increasing demand, ensuring the continued success of this innovative program.

\section{Acknowledgements}

The authors thank Connie Winther at the Kaye Edmonton Clinic, for all of her efforts, contributions, and dedication as co-lead of the KRS Research and Reference work group.

\section{References}

1. Alberta Health Services (AHS). Our history. 2010 [cited 3 Aug 2014]. Available from: http://www.albertahealthservices.ca/ 191.asp

2. Alberta Health Services. Alberta Health Services Health Plan and Business Plan (2012-2015) [Internet]. 2012 Apr [cited 8 Jul 2014]. Available from: http://www.albertahealthservices. ca/Publications/ahs-pub-2012-2015-health-plan.pdf

3. Stieda V, Colvin B. Descriptive analysis of the inequalities of health information resources between Alberta's rural and urban health regions. Healthc. Q. 2009;12(3):66-70. doi: 10.12927/hcq.2013.20880.

4. Pomerantz J, Nicholson S, Lankes RD. Digital reference triage: Factors influencing question routing and assignment. Lib. Q. 2003;73(2):103-120. doi: 10.1086/603392.

5. Lyon JA, Garcia-Milian R, Norton HF, Tennant MR. The use of Research Electronic Data Capture (REDCap) software to create a database of librarian-mediated literature searches. Med. Ref. Serv. Q. 2014;33(3):241-252. doi: 10.1080/02763869. 2014.925379.

6. Finfgeld-Connett D, Johnson ED. Literature search strategies for conducting knowledge-building and theory-generating qualitative systematic reviews. J. Adv. Nurs. 2012;69(1):194204. doi: 10.1111/j.1365-2648.2012.06037.x. 